\title{
Absolute declinations with the photoelectric astrolabe at Calern Observatory (OCA)^
}

\author{
V.A.F. Martin ${ }^{1,2}$, N.V. Leister ${ }^{3}$, G. Vigouroux ${ }^{4}$, M. Furia ${ }^{4}$, and A. Journet ${ }^{4}$ \\ 1 Observatório Astronômico Antares, Universidade Estadual de Feira de Santana, Rua da Barra, 925, cep: 44015-430, Feira de \\ Santana, Bahia, Brazil \\ 2 Departamento de Física, Universidade Estadual de Feira de Santana, Rodovia BR116 Norte, km 03, Campus Universitário, \\ cep: 44031-460, Feira de Santana, Bahia, Brazil \\ 3 Instituto Astronômico e Geofísico, Universidade de São Paulo, Av. Miguel Stéfano, 4200, cep: 04301-970, São Paulo SP, Brazil \\ ${ }^{4}$ Observatoire de la Côte d'Azur, C.E.R.G.A., avenue Copernic, F-06130 Grasse, France
}

Received September 22; accepted March 18, 1999

\begin{abstract}
A regular observational programme with a photoelectric astrolabe have been performed at "Observatoire du Calern" (Observatoire de la Côte d'Azur, OCA, $\phi=+43^{\circ} 44^{\prime} 55.011^{\prime \prime} ; \lambda=-0^{\mathrm{h}} 27^{\mathrm{m}} 42.44^{\mathrm{s}}$, Calern, Caussols, France) for the last twenty years. It has been almost fully automatized between 1984 and 1987. Since 1988 the photoelectric astrolabe was used without any modification. In addition to determining the daily orientation of the local vertical, the yearly analysis of the residuals permits to derive corrections to the used star catalogue (Vigouroux et al. 1992).

A global reduction method was applied for the ASPHO observations. The new form of the equations (Martin \& Leister 1997) give us the possibility of using the entire set of the observing program using data taken at two zenith distances $\left(30^{\circ}\right.$ and $\left.45^{\circ}\right)$.

The program contains about 41648 stars' transits of 269 different stars taken at "Observatoire du Calern" (OCA).

The reduction was based on the HIPPARCOS system. We discuss the possibility of computing absolute declinations through stars belonging simultaneously to the $30^{\circ}$ and $45^{\circ}$ zenith distances programmes. The absolute declination corrections were determined for 185 stars with precision of $0.027^{\prime \prime}$ and the value of the determined equator correction is $-0.018^{\prime \prime} \pm 0.005^{\prime \prime}$. The instrumental effects were also determined. The mean epoch is 1995.29.
\end{abstract}

Key words: astrometry — catalogues — reference system

Send offprint requests to: V.A.F. Martin,

e-mail: vmartin@uefs.br

* Catalogue only available at CDS in electronic from via anonymous ftp to cdsarc.u-strasbg.fr (130.79.128.5) or via http://cdsweb.u-strasbg.fr/Abstract.html

\section{Introduction}

Although the HIPPARCOS system will make remarkable achievements, there are many things to do after HIPPARCOS (Clauzet et al. 1990; Kovalevsky 1991), such as: the improvement of fundamental reference systems of stars, the extension of fundamental catalogue to faint stars, the maintenance of the HIPPARCOS reference system, the extension of HIPPARCOS catalogue.

The automatized photoelectric astrolabe (here-after ASPHO) at the "Observatoire du Calern" (OCA, $\phi=$ $+43^{\mathrm{o}} 44^{\prime} 55.011^{\prime \prime} ; \lambda=-0^{\mathrm{h}} 27^{\mathrm{m}} 42.44^{\mathrm{s}}$, Calern, Caussols, France) observed two years at two zenith distances $\left(30^{\circ}\right.$ and $45^{\circ}$ ) which turns it able to determine absolute declinations of stars (Martin \& Leister 1997). One must note that automatization not only gave to ASPHO a real impersonality, but also a very much improved output, particularly during the long winter nights.

After July 1993, the pillar made in concret was replaced by a crude metalic pillar. Thus, the thermal problems in the ASPHO were diminished and was possible to put an elevator to the mercury basin. This made possible the observations at $30^{\circ}$ and $45^{\circ}$ zenithal distances.

The stars are arranged in two different observational programmes (ASPHOI and ASPHOII), in 11 groups of fixed composition with the best azimuth repartition.

In the period 1993-1996, 41648 stars transits were observed in the declination zone $-2^{\circ}$ to $88^{\circ}$. The catalogues composition are 169 stars at ASPHOI $\left(30^{\circ}\right)$ and 180 at ASPHOII $\left(45^{\circ}\right)$ having a total of 349 stars.

In this total was included 84 stars at almost maxima digression condition and 62 that were common at the two catalogues.

With this set of data it was possible to determine 185 absolute declinations $\left(\delta_{\mathrm{abs}}\right)$. Our method allows to obtain the declination corrections of all stars. 
In this paper we consider all the programme stars in the global reduction method (Martin et al. 1996; Martin \& Leister 1997). We accomplished the global reduction due the small number of common stars in the catalogues observed at two zenith distances.

Despite the fact that the number of stars is small, this method allowed a better evaluation of the observational set. We obtained a better definition of the errors involved in the process and a global definition of the colour and magnitude equations; furthermore we were able to get directly the equator correction $(\eta)$ and the declination correction $(\Delta \delta)$.

\section{Observational method}

The observational programme $\left(30^{\circ}\right.$ or $\left.45^{\circ}\right)$ was changed every 3 or 4 weeks. All the star groups must be sufficiently observed to make the internal and the external agreements and to have the significative mean residuals.

At Calern Observatory there are near 200 nights of observation per year that allow to obtain more or less 600 groups. In summer this quantity is smaller ( 2 or 3 groups) but the observational quality is better. The autocolimation is used to position the optical square with precision.

\section{Reduction method}

We have one conditional equation for each observation:

$(\mathrm{O}-\mathrm{C})(-\sin Z \cdot \cos \phi)=$

$=1 / 15[(\mathrm{UT} 0-\mathrm{UTC})-\Delta L] \sin Z \cdot \cos \phi+\Delta \phi \cdot \cos Z+R$

$-\Delta \alpha \cdot \sin Z \cdot \cos \phi-\Delta \delta \cdot \cos S$

where: UT0 - UTC, $\Delta L$ and $\Delta \phi$ represent the deviations related to the pole motion and the Earth rotation velocity; $R$ is related to the zenith distance correction.

We obtain the residual $(\mathrm{O}-\mathrm{C})$ after the groups reduction for each star passage (east and west).

\section{Analysis method}

A global reduction method was applied for photoelectric astrolabe residuals. The approach is much simpler although more precise than the conventional method. The new form of the equations (Martin et al. 1996; Martin \& Leister 1997) give us the possibility of using the entire set of the observing programme using the different data taken at two zenith distances.

In this method, the data and the unknowns are treated as symmetrically as possible with a determined statistical weighting. The used symmetric form aims to get the mean residuals with statistical independence and uniform variance.
Considering all the star observations $(i)$ in the involved catalogues $(j)$, we can write, for each transit (east and west - E and W), the following equations (Martin \& Leister 1997):

$$
\begin{aligned}
-R_{i \mathrm{E}}^{j}= & -\Delta \alpha_{i}\left|\sin Z_{i \mathrm{E}}^{j}\right| \cos \phi+\Delta \delta_{i} \cos S_{i}^{j}+ \\
& +\xi^{j}\left|\sin Z_{i \mathrm{E}}^{j}\right|+\eta^{j} \cos Z_{i \mathrm{E}}^{j}-\zeta^{j} \\
-R_{i \mathrm{~W}}^{j}= & +\Delta \alpha_{i}\left|\sin Z_{i \mathrm{~W}}^{j}\right| \cos \phi+\Delta \delta_{i} \cos S_{i}^{j}+ \\
& -\xi^{j}\left|\sin Z_{i \mathrm{~W}}^{j}\right|+\eta^{j} \cos Z_{i \mathrm{~W}}^{j}-\zeta^{j}
\end{aligned}
$$

where:

$R_{\mathrm{E}}$ and $R_{\mathrm{W}}$ are the east and west mean residuals in zenith distance after the addition of group corrections for east and west transits of the star (homogenisation of the stellar groups), and $S$ is the parallactic angle. The constant $\eta$ can be understood as the difference between the real and observed values of the latitude after the homogenisation of the stellar groups (related with the equator correction); the constant $\zeta$ is related to the true zenith distance of observation (instrumental effects); $Z$ is the star azimuth from North to East; $\phi$ the local latitude, and $\xi$ the constant related to the equinox correction.

Adding the mean residuals $\left(R_{\mathrm{E}}\right.$ and $\left.R_{\mathrm{W}}\right)$ above, the stellar declination corrections can be computed from astrolabe observations by the formula (Débarbat \& Guinot 1970):

$$
\begin{aligned}
\Delta \delta_{i}= & -\left[\left(R_{\mathrm{E}}+R_{\mathrm{W}}\right)\right]_{i}^{j} /\left(2 \cos S_{i}^{j}\right)-\eta^{j} \cos Z_{i}^{j} / \cos S_{i}^{j}+ \\
& +\zeta^{j} / \cos S_{i}^{j}
\end{aligned}
$$

From Eq. (3), we can write:

$\left[\left(R_{\mathrm{E}}+R_{\mathrm{W}}\right) / 2\right]_{i}^{j}=-\Delta \delta_{i} \cos S_{i}^{j}-\eta^{j} \cos Z_{i}^{j}+\zeta^{j}$.

Considering all of the star observations with double passage $(\mathrm{E} / \mathrm{W})$, and the colour index and magnitude function $C\left(I_{i}, m_{i}\right)^{1}$, we get the average of the mean residuals $\left(\rho_{i}^{j}\right)$ :

$$
\begin{aligned}
\rho_{i}^{j}= & -\Delta \delta_{i} \cos S_{i}^{j}+\left(B_{1} m_{i}+B_{2} m_{i}^{2}+A_{1} I_{i}\right. \\
& +\Delta z)^{j}-\eta^{j} \cos Z_{i}^{j},
\end{aligned}
$$

where:

$\rho=\left(R_{\mathrm{E}}+R_{\mathrm{W}}\right) / 2 ; B_{1}, B_{2}, A_{1}$ are the magnitude and colour index coefficients; $m_{i}$ is the visual apparent magnitude of the star; $I_{i}$ is the visual colour index $(B-V)$ of the star and $\Delta z$ is the zenith distance correction.

The possibility of computing absolute declinations rests on the determination of $\eta$.

This equation can be applied to all stars even to transit stars with only one zenith distance to get systematic differences $\Delta \delta$, which is not possible with the classical methods. For the stars which are at maximum digression condition

\footnotetext{
1 Polynomial function: second degree to magnitude and first degree to colour index - (Boczko 1989; Martin \& Clauzet 1990; Martin et al. 1996; Martin 1998).
} 
Table 1. Colour and magnitude coefficients - (global reduction)

\begin{tabular}{lll}
\hline & & catalogues \\
\hline & ASPHO1 & ASPHO2 \\
\hline$B_{1}\left({ }^{\prime \prime}\right)$ & $-0.007 \pm 0.011$ & $-0.016 \pm 0.011$ \\
$B_{2}\left(^{\prime \prime}\right)$ & $+0.015 \pm 0.025$ & $+0.025 \pm 0.029$ \\
$A_{1}\left({ }^{\prime \prime}\right)$ & $-0.001 \pm 0.012$ & $+0.009 \pm 0.010$ \\
$\Delta z\left(^{\prime \prime}\right)$ & $-0.009 \pm 0.003$ & $-0.007 \pm 0.009$ \\
\hline
\end{tabular}

the coefficients of $\cos S$ in the matrix vanishes, and these stars do not contribute to the $\Delta \delta$ determinations.

However, the system has a singularity, which is removed by the condition:

$\eta_{\mathrm{ASPHO} 1}=\eta_{\mathrm{ASPHO} 2}=\eta$.

\section{Results}

We used 269 different stars belonging to ASPHO1 and ASPHO2 catalogues obtained at OCA. Our goal is to determine 185 declination corrections $(\Delta \delta)$ taking into account that 84 stars were in the near maxima digression condition.

An analysis of the individual values of $\Delta \delta$ as obtained from global reduction as well as the extension to the noncommon stars, was made and the results are based on HIPPARCOS system.

The $\Delta \delta$ are obtained as the addition of the east and west mean residuals that means they may contain instrumental effects such as magnitude and colour equations. In Table 1 we have the magnitude and colour index coefficients (instrumental effects) obtained by the global reduction.

Based on Table 1 we can conclude that the colourmagnitude effects in the photoelectric astrolabe (ASPHO) are very small confirming the quality of the results.

These results are comparable with the best results presented in the literature. The general standard deviation obtained by the global reduction is $0.027^{\prime \prime}$ and the value of $\eta$ is $-0.018^{\prime \prime} \pm 0.005^{\prime \prime}$.

It is interesting to note that the magnitudes analysed here are not bigger than 8.0. Thus, we can only say that there is not any colour-magnitude effect in this term. It is possible that it is not true to magnitudes bigger than 8.0. There is a project to transform the detection from photoelectric to CCD form.

The obtained equator correction is small confirming that the equator used in the FK5 system in the observed zone as well as that these values are compatible with the dynamical values for the equator correction taking with others techniques (Leister 1989) as showed in Fig. 1.

The comparison of our results with the dynamical ones confirms the astrolabe as a potential instrument for ground-based astrometry.

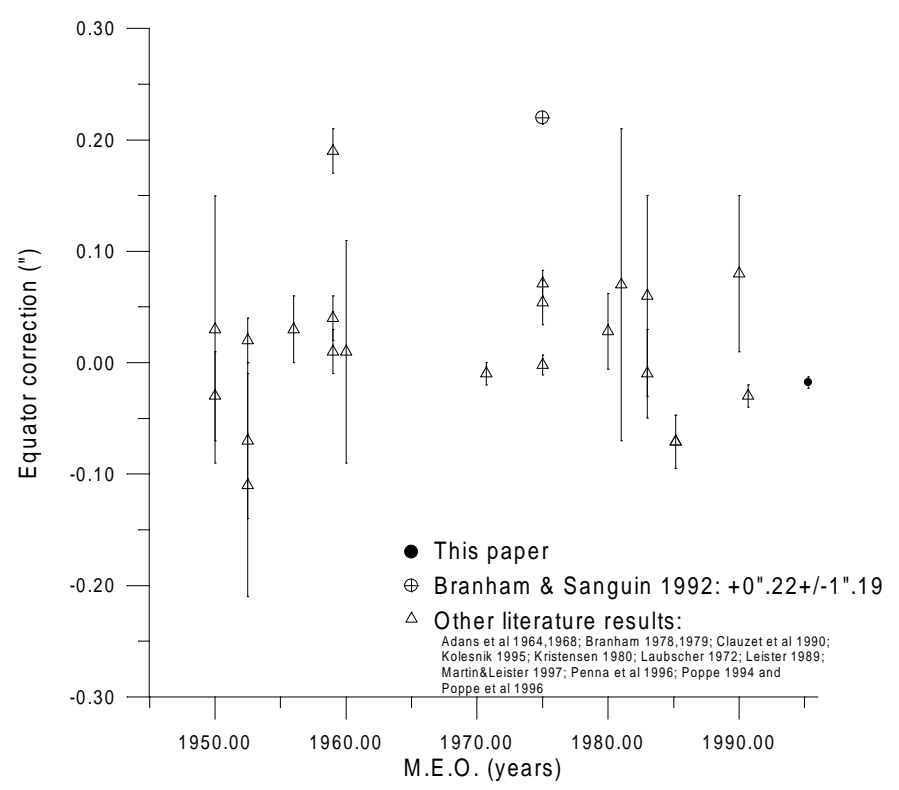

Fig. 1. Equator corrections (Martin \& Leister 1997, modified)

Thus, the photoelectric astrolabe can be a very important instrument for the definition of the fundamental reference equator. The results confirm also the position adopted by Fricke (1982) in order to maintain the equator of the FK4 system in the FK5 system.

The $\Delta \delta$ and $\sigma_{\Delta \delta}$ are shown in Figs. 2a,b. The $\Delta \delta$ distribution has mean $-0.010^{\prime \prime}$ and standard deviation $0.003^{\prime \prime}$ and the $\sigma_{\Delta \delta}$ distribution has mean $0.049^{\prime \prime}$ and standard deviation $0.003^{\prime \prime}$. The dotted line represents the Gaussian fitting.

\section{Discussion}

It must be pointed out that, in our case, we estimated the equator correction and $\Delta \delta$ corrections for the declination zone (mostly north hemisphere) of the ASPHO observations $\left(-2^{\circ} \leq \delta \leq 88^{\circ}\right)$.

In a near future we will reduce all the data taking into account the IERS system utilizing the $\Delta \phi$ and $\Delta t$ given by the IERS and the $\Delta R$ given by the group observations. We expect to obtain results not so different from these ones but with a better precision.

\section{Conclusions}

In spite of the small number of common stars in ASPHO1 and ASPHO2 catalogues, the results confirm the opportunity and validity of the global reduction to compute absolute declinations. The comparison of our results with dynamical ones confirms the astrolabe as a potential instrument for this class of observations.

Since February 1998, the photoelectric astrolabe from Calern Observatory (France) is at Antares Astronomical 


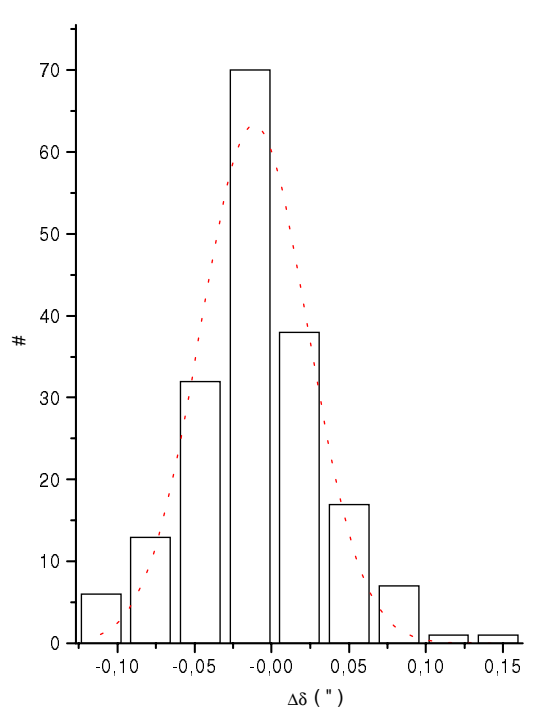

Fig. 2. a) $\Delta \delta$ and b) $\sigma_{\Delta \delta}$ distribution

Observatory $\left(\lambda=+38^{\circ} 57^{\prime} 53^{\prime \prime} ; \phi=-12^{\circ} 15^{\prime} 25^{\prime \prime}\right.$, Bahia, Brazil). We hope, in a near future, to present the results of the observations that will start on March 1999.

Acknowledgements. The authors wish to thank the FAST consortium for the HIPPARCOS data. We are also grateful to R. Boche (in memorian) and B. Chauvineau for their participation in the observational programme. The authors are also grateful to G. Billaud for the conception of the ASPHO. He has guided the team at Calern Observatory until he retired on September 1990. Financial support from CNPq and FAPESP No. 92/3762-0 is gratefully acknowledged.

\section{References}

Adans A.N., Bestul S.M., Scott D.K., 1964, Publ. U.S. Naval Obs. 19, p. 1

Adans A.N., Scott D.K., 1968, Publ. U.S. Naval Obs. 19, p. 1 and 2

Boczko R., 1989, PhD Thesis, IAGUSP, Brazil

Branham Jr. R.L., 1978, AJ 83, 675-681

Branham Jr. R.L., 1979, AJ 84, 1399-1401

Branham Jr. R.L., Sanguin J.G., 1992, AJ 103, No. 6, 2099

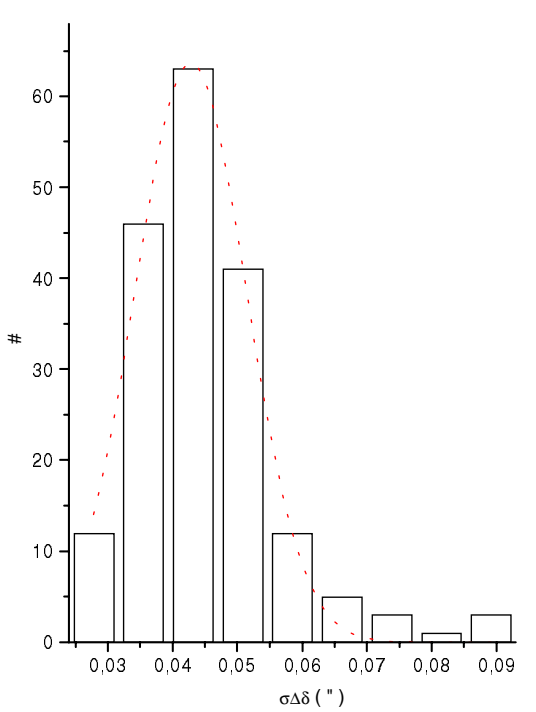

Clauzet L.B.F., et al., 1990, in: Colloque A. Danjon, Journées SRST, Capitaine N. (eds.). Paris, p. 89

Débarbat S., Guinot B., 1970, La méthode des hauteurs égales en astronomie. Gordon \& Breach (eds.), Paris

van Flandern T.C., 1971a, Celest. Mech. 4, 182

van Flandern T.C., 1971b, AJ 76, 81-82

Fricke W., 1982, A\&A 107, L13-L14

Kolesnik Y.B., 1995, A\&A 294, 874-894

Kovalevsky J., 1991, Astrophys. Space Sci. 177, 457

Kristensen L.K., 1980, Mitteil. Astron. Gesellschaft, 48

Laubscher R.E., 1972, A\&A 20, 407-414

Leister N.V., 1989, PhD. Thesis, IAGUSP, Brazil

Martin V.A.F., Clauzet L.B.F., 1990, Rev. Mex. Astron. Astrof., 21, 297-299

Martin V.A.F., Boczko R., Benevides-Soares P., Leister N.V., 1996, A\&AS 118, 525-528

Martin V.A.F., Leister N.V., 1997, A\&AS 126, 169-176

Martin V.A.F., 1998, PhD. Thesis, IAGUSP, Brazil

Penna J.L., Benevides-Soares P., Andrei A.H., Laclare F., Leister N.V., 1996, A\&A 310, 1036

Poppe P.C.R., 1994, Msc. Thesis, IAGUSP, Brazil

Poppe P.C.R., Leister N.V., Laclare F., 1996, A\&A 315, 633

Vigouroux G., Billaud G., Boche R., Chauvineau B., Furia M., Journet A., Kovalevsky J., 1992, A\&AS 96, 477-484 